\title{
New animal model for human ocular toxocariasis: ophthalmoscopic observation
}

Tomoko Hara Takayanagi, Nobuaki Akao, Ryoko Suzuki, Misato Tomoda, Setsuko Tsukidate, Koichiro Fujita

\begin{abstract}
Backgroundlaims-Although human ocular toxocariasis causes severe vision defect, little is known about its aetiology, diagnosis, and treatment. To develop a new animal model for human ocular toxocariasis, ophthalmological findings of fundi in Mongolian gerbils, Meriones unguiculatus, and BALB/c mice were investigated following infection with Toxocara canis.

Methods-Using an ophthalmoscope, which was specifically developed to observe the fundi of small animals, ocular changes of fundi of 20 gerbils and 11 mice were monitored after oral infection with embryonated eggs of $T$ canis.

Results-Vitreous, choroidal, and retinal haemorrhages were consistently observed in Mongolian gerbils, but rarely in mice. Severe exudative lesions and vasculitis were often present in gerbils but not in mice. Migrating larvae were also frequently observed in gerbils.

Conclusion-Mongolian gerbils are more appropriate animal model for human ocular toxocariasis than previously used experimental animal such as mice, guinea pigs, rabbits, and monkeys because of its high susceptibility of ocular infection.

(Br F Ophthalmol 1999;83:967-972)
\end{abstract}

Ocular nematodes were first observed under the microscope in $1950^{1}$ during pathological examinations of enucleated eyes. Nichols reported those were the second stage larvae of Toxocara canis. ${ }^{2}$ Since then, many cases of ocular toxocariasis have been reported from around the world. ${ }^{3-7}$ Ocular toxocariasis was classified by Wilkinson and Welch in 1971 as (1) diffuse nematode endopththalmitis, (2) posterior pole granuloma, and (3) peripheral inflammatory mass. ${ }^{8}$ Little is known about the aetiology of the ocular changes, the route of migration to the eyes, diagnosis, treatment, and prognosis of this disease. Diagnoses of ocular toxocariasis had been made retrospectively, by detecting larva in enucleated eyes ${ }^{3-7}$ until the time Wilkinson and Welch made their classification. There has been only one case, reported by Maguire et al, in which a definite diagnosis of ocular toxocariasis could be made without enucleation by detecting larva in recovery from vitrectomy. ${ }^{9}$ Serological examinations were not done when Wilkinson made the classifications, which means there might be some cases in which ocular toxocariasis was misdiagnosed. Clinical diagnosis is now usually done ophthal- moscopically and serologically by enzyme linked immunosorbent assay (ELISA) with excretory-secretory (ES) antigen. However, the diagnostic titre remains controversial. ${ }^{10}$ Watzke described the case of an enucleated eye which showed larva, but was negative for serum antibody. ${ }^{11}$ Several cases have been reported as having a low ELISA titre to Toxocara canis. ${ }^{12-14}$ Vitreous fluid and aqueous humour are sometimes evaluated by ELISA, ${ }^{15} 16$ as well as serum, and aqueous humour is said to have greater diagnostic accuracy than serum. However, taking samples of aqueous humour involves an invasive procedure and so it is not a common examination.

Even if a diagnosis can be made there are no effective treatments at present. Oral anthelmintics, ${ }^{16}$ oral steroid, ${ }^{16}{ }^{17}$ photocoagulation, ${ }^{18-21}$ vitrectomy, ${ }^{922} 23$ and cryoretinopexy ${ }^{2425}$ have been tried, but with unsatisfactory results. Dead larvae reportedly can cause a much severer reaction in the host, ${ }^{26}$ so it is not yet known even whether or not killing the larva will be therapeutic. ${ }^{26}$ In order to develop practical and precise new methods of diagnosis, to understand the aetiology of the ocular changes caused by Toxocara infection, and to conduct trials of each type of treatment, experimental animals which accurately reflect human ocular toxocariasis are needed.

Mice, ${ }^{27-29}$ rabbits, ${ }^{28}$ guinea pigs, ${ }^{30} 31$ and monkeys ${ }^{32}{ }^{33}$ had been evaluated pathologically for use as an experimental animal model for ocular toxocariasis. With these animals, however, the incidence of ocular infection is low and eosinophilic infiltration is rarely observed. Furthermore, we believe that ophthalmoscopic observation is important in order to observe the clinical course of ocular infection, but to our knowledge only one case has been reported in which motile larvae have been identified in the retina of one of these animals, an intravitreal infected monkey. ${ }^{32}$ A suitable experimental animal model should be highly susceptible to ocular infection and exhibit various abnormalities.

Mongolian gerbils are known to be susceptible to a variety of parasites including Brugia pahangi, ${ }^{34}$ Strongyloides stercoralis, ${ }^{35}$ Nippostrongylus brasiliensis, ${ }^{35}$ and Entamoeba hystolytica. ${ }^{36}$ In the present study, we carried out oral inoculation of Toxocara canis in Mongolian gerbils and report their usefulness as a new animal model for human ocular toxocariasis.

\section{Materials and methods}

ANIMALS

We used 11 five week old female BALB/c mice and 20 three to four month old female Mongo- 

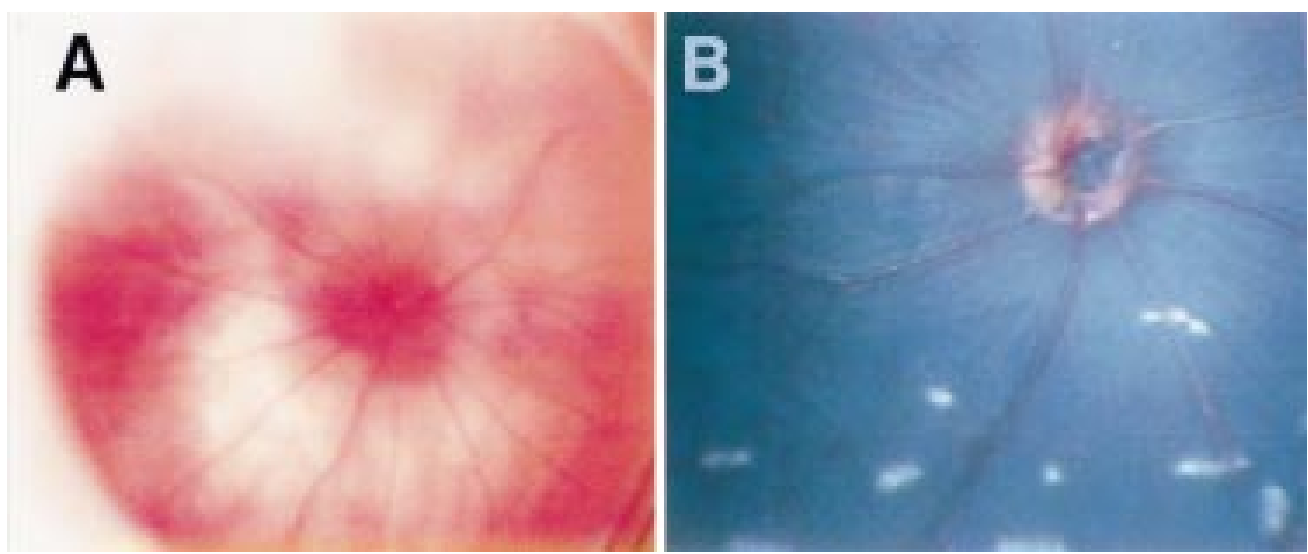

Figure 1 Normal fundus of BALB/c mouse and Mongolian gerbils. (A) Normal fundus of BALB/c mouse. (B) Normal fundus of Mongolian gerbils. Rod-shaped multiple white spots were seen in normal fundus of gerbils.

lian gerbils, Meriones unguiculatus. Mice were purchased from Charles River Japan, Inc (Yokohama, Japan) and Mongolian gerbils were raised in our laboratory. Both types of animals have been maintained under specific pathogen-free conditions in the Animal Centre of Tokyo Medical and Dental University.

\section{PARASITE AND INFECTION}

Embryonated eggs of $T$ canis were prepared following the method of Oshima, ${ }^{37}$ and 17 eggs/g body weight were inoculated into each animal via gastric tube under light anaesthesia. The number of eggs was decided by our preliminary graded inoculation experiment and Oshima's method. ${ }^{37}$ All equipment was siliconised for the complete administration of the designated number of eggs.

\section{OCULAR OBSERVATION}

Both groups of animals were anaesthetised with sodium pentobarbitone (30 $\mathrm{mg} / \mathrm{kg}$, Pittoman-Moore, NJ, USA), and their pupils were dilated with tropicamide (one drop for each eye, Mydrin-P, Santen Pharmaceutical Co Ltd, Japan). Ocular changes in each animal were observed with an ophthalmoscope (Scalar VMS-170M, Abbe Science, Japan). This ophthalmoscope was specifically adapted for observing fundi of small animals. The images of the fundi were transferred to a TV monitor for viewing, and recorded on videotape. Both groups of animals were observed from day 0 until the end of the designated period- -78 days after inoculation for mice, 158 days for gerbils.

PATHOLOGICAL EXAMINATION

Both groups of animals were sacrificed at the end of the designated period using sodium pentobarbitone. Eyeballs were enucleated im-

Table 1 Abnormal ocular findings in Mongolian gerbils and BALB/c mice

\begin{tabular}{|c|c|c|c|c|}
\hline Abnormal findings & $\begin{array}{l}\text { No of gerbils } \\
(\%)\end{array}$ & $\begin{array}{l}\text { No of eyes } \\
(\%)\end{array}$ & $\begin{array}{l}\text { No of mice } \\
(\%)\end{array}$ & $\begin{array}{l}\text { No of eyes } \\
(\%)\end{array}$ \\
\hline \multicolumn{5}{|l|}{ Haemorrhagic changes } \\
\hline choroidal haemorrhage & $19(95.0)$ & $30(75.0)$ & - & - \\
\hline retinal haemorrhage & $11(55.0)$ & $16(40.0)$ & - & - \\
\hline white centred retinal haemorrhage & $9(45.0)$ & $12(30.0)$ & $8(72.7)$ & $9(40.9)$ \\
\hline vitreous haemorrhage & $1(5.0)$ & $1(2.5)$ & - & - \\
\hline Exudative lesion & $15(70.0)$ & $27(67.5)$ & - & - \\
\hline Vasculitis & $10(25.0)$ & $11(27.5)$ & - & - \\
\hline Larva & $16(80.0)$ & $26(65.0)$ & - & - \\
\hline
\end{tabular}

mediately after the animals died and fixed in $2.5 \%$ glutaraldehyde and $4 \%$ formaldehyde in $0.15 \%$ phosphate buffer $(\mathrm{pH} 7.2)$. Serial section were taken and stained with haematoxylin and eosin and periodic acid Schiff haematoxylin.

\section{CONTROL GROUP}

Three mice and one gerbil were anaesthetised and their fundi observed following the same procedure without inoculation. Control animals were observed from day 0 to day 7 . None of them showed abnormal changes.

\section{Results}

NORMAL FUNDI OF BALB/C MICE AND MONGOLIAN GERBILS

Papilla and vessels of fundi of BALB/c mice can be seen clearly with an ophthalmoscope, but because these fundi are albinotic, larvae and exudative lesions cannot be detected (Fig 1A). During the observation, the eyes easily dried out and corneas turn cloudy making fundi difficult to observe. With the gerbils, not only papilla and vessels, but also retinal patterns, were observable with the ophthalmoscope and there were no problems with the corneas. Rod-shaped multiple white spots were observed in the normal fundi of the gerbils (Fig 1B). The macular area was not obvious in either animal.

INCIDENCE OF THE OCULAR LESIONS

Table 1 showed the incidence of the ocular lesions in gerbils and mice. In gerbils, we found haemorrhagic lesions, exudative lesions, vitreous opacity, and abnormality of vessels with migrating larvae after 3 days of infection. However, only one type of haemorrhagic lesion was found in eight of the 11 mice by day 10 , and no exudative lesions or migrating larvae were detected. Mice were inoculated again on day 30 with same number of embryonated eggs and observed until day 78. No new lesions were seen after the second inoculation. Only one gerbil had no abnormal ocular abnormalities. Bilateral abnormalities were present in 17 gerbils, and unilateral abnormalities in one.

HAEMORRHAGIC LESIONS (TABLE 1)

Four types of haemorrhagic changes were seen in gerbils' fundi. Vitreous haemorrhage was 

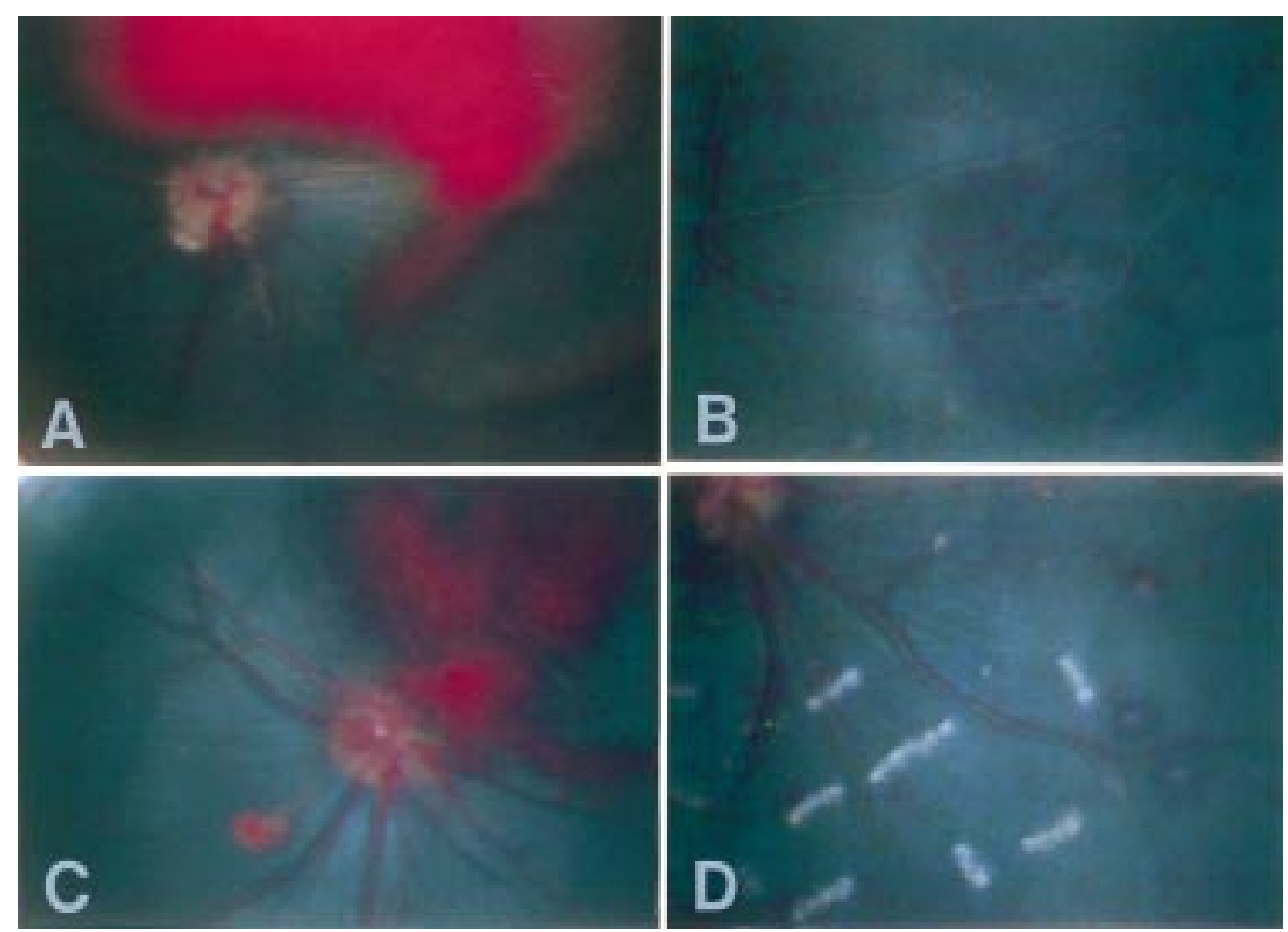

Figure 2 Haemorrhagic changes. (A) Vitreous haemorrhage-was seen in two of the 20 gerbils from day 7 after infection of $T$ canis. This was associated with vitreous opacity and was absorbed within 1 month. (B) Choroidal haemorrhage-was found in $95 \%$ of gerbils with a single oral inoculation of $T$ canis embryonated eggs. (C) Retinal haemorrhage-was seen in $55 \%$ of $T$ canis infected gerbils. Large central retinal haemorrhage was observed. (D) White centred retinal haemorrhage. Multiple small haemorrhage with a white central part were found in the periphery.

seen in two of the 20 gerbils from day 7 . This was associated with vitreous opacity and was absorbed within 1 month (Fig 2A). Choroidal haemorrhages were the most frequent; $95 \%$ of gerbils had this type of haemorrhage from day 3 and recurrences were observed until a maximum of day 133. Most were detected in the peripheral region (Fig 2B). Retinal haemorrhages were found in $55 \%$ of gerbils between day 3 and day 98. Large haemorrhages were observed around the optic nerve (Fig 2C) in two gerbils. Multiple, small round haemorrhages with white centres (Fig 2D) were observed from day 5 to day 135 .

\section{EXUDATIVE LESIONS}

Exudative lesions which were white or yellowish, with soft or hard patches, and irregular colour changes of the retina suggestive of cho-

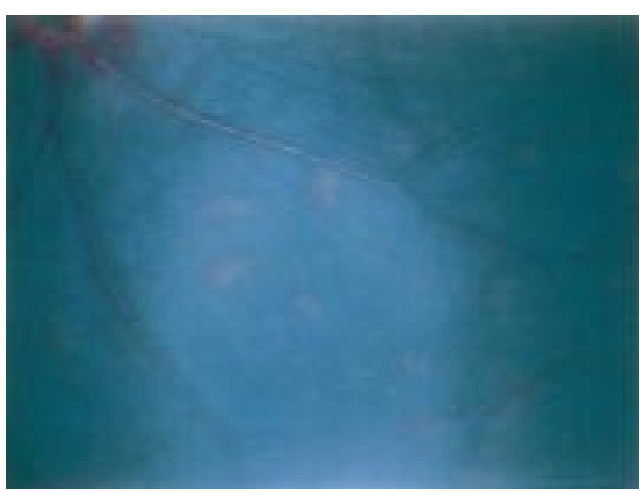

Figure 3 Exudative lesion. Irregular colour changes of retinal pattern and soft or hard patches suggested chorioretinitis in $70 \%$ of gerbils with oral inoculation of $T$ canis. rioretinitis were detected in $75 \%$ of gerbils between day 3 and the end of the observation period (Table 1 and Fig 3). The latest occurrence of exudative lesions was from day 103. These did not disappear once they occurred.

\section{VESSEL ABNORMALITY}

Narrowing, dilatation, tortuosity (Fig 4A) of vessels was found in one gerbil from day 7 . White exudative lesions around the vessel walls that may suggest vasculitis were seen mostly near bifurcation (Fig 4B) in nine gerbils from day 3 to day 55 at the latest. Indeed, pathological examination revealed that cell infiltration around vessels, especially eosinophils and lymphocytes, was a predominant feature (Fig 4C), indicating vasculitis was induced by the infection. These did not disappear once they occurred.

MIGRATING LARVAE

We found migrating larvae in $80 \%$ of gerbils from day 3 and they were present until the end of the observation period. A maximum of three migrating larvae was seen in one eye. The larvae migrated on the retina freely. We could see them migrating over and under the vessel (Fig 5). The white larvae were easily detectable in the gerbils' dark grey fundi.

LARVAE TRACES

A few larvae left traces on the retina (Fig 6). Traces were made just after the larva migrated to another site. The yellow whitish bright restiform traces were observed in real time when larvae were migrating. However, these larvae 

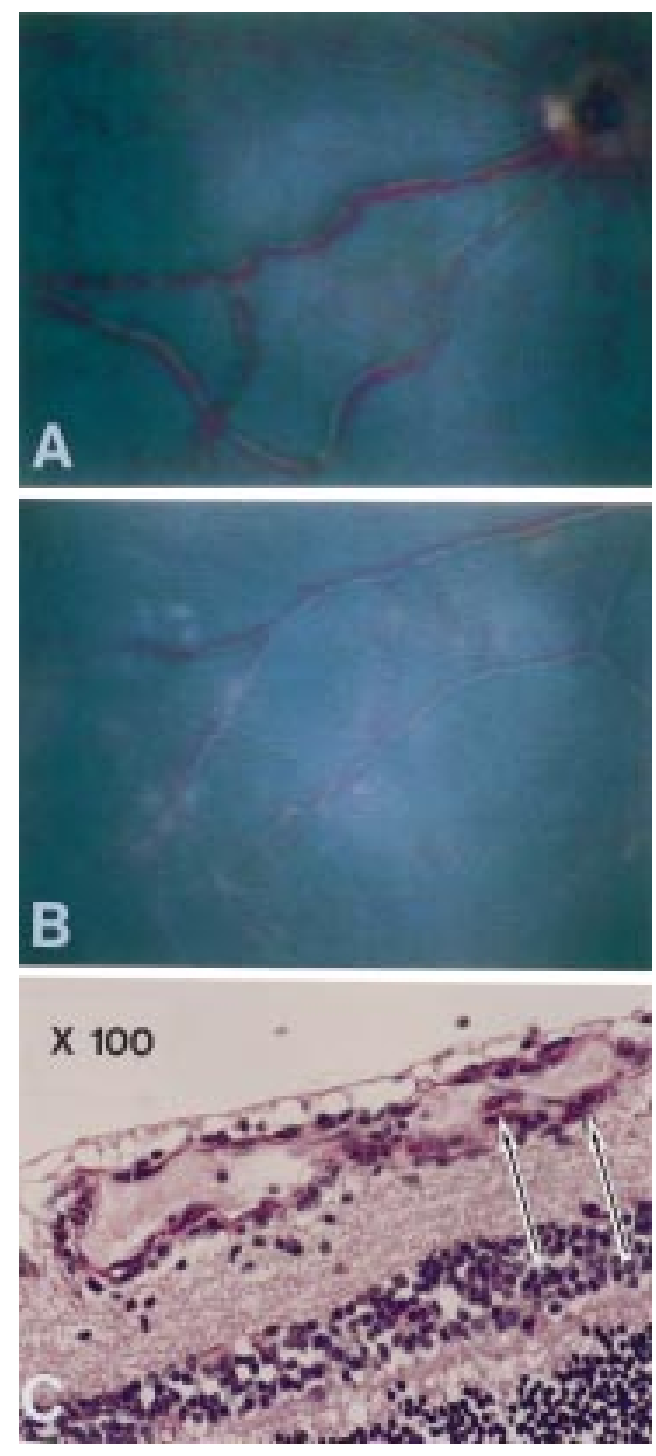

Figure 4 Vasculitis. (A) Tortuosity, dilatation, and narrowing of vessels. (B) White exudative lesion around the bifurcation of vessels. (C) Eosinophils (arrows) and lymphocyte infiltration were seen on the vessel wall.

did not always leave traces. These traces sometimes ended during migration, and sometimes lasted over the entire migration. Only two traces were observed clearly in our whole observation. One disappeared in 2 days, and the other disappeared within 20 days.

NATURAL COURSE OF OCULAR INFECTION IN GERBILS

All the abnormal findings started on day 3. The first abnormal findings were haemorrhagic changes and migrating larvae. Haemorrhagic changes, especially choroidal haemorrhage, recurred several times in the same eyes until the end of the observation period. Larvae were found from day 3 through to the end of the observation period, the last one appearing on day 126. They could be seen in almost the same region on the surface or in the retinal layer in most eyes, but sometimes moved rapidly from around the papilla to the periphery. Although we could not directly observe larvae perforating vessels or causing haemorrhage, all eyes in which one or more larvae were

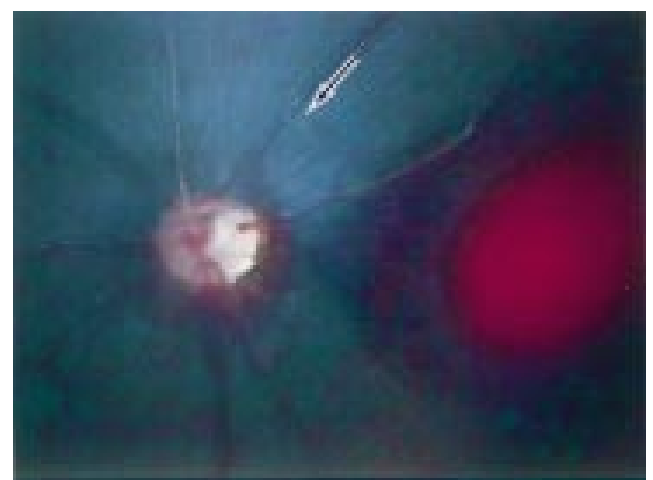

Figure 5 Motile larvae of T canis. Larva (arrow) can migrate vigorously in the retinal layer. Larvae could be found in $80 \%$ of gerbils.

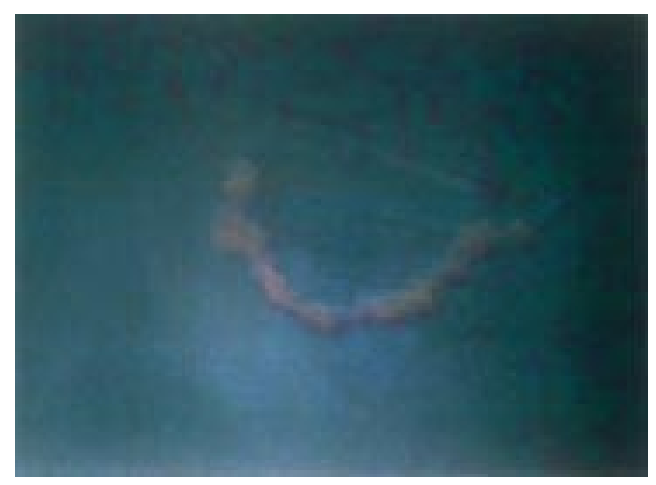

Figure 6 Trace of larvae of $T$ canis. This trace disappeared within 20 days.

found had haemorrhagic lesions. Haemorrhagic lesions were absorbed within 1 month with fibrin or hard yellowish patches persisting after the haemorrhages. Exudative lesions and vasculitis were chronic changes that did not disappear throughout the observation period once they occurred. No granulomatous lesion or retinal detachment was detected in any of the animals.

\section{Discussion}

In this study we found that Mongolian gerbils have a high susceptibility to ocular infection by $T$ canis. Severe ocular changes such as large haemorrhage, exudative lesions, and perivasculitis were frequently observed following a single oral inoculation. In addition, the dark grey fundi of these animals make them very suitable for observations of the motile white larvae of $T$ canis. In contrast, ophthalmological changes were hard to detect in BALB/c mice and larvae were difficult to identify because of the albino fundi of these mice. Moreover, the corneas of mice had a strong tendency to dry out during observation, and consequently were permanently clouded, making it difficult for us to continue our experiment. Therefore, even if we used another strain of mice with pigmented retinas, such as $\mathrm{C} 57 \mathrm{BL}$ and $\mathrm{C} 3 \mathrm{H} / \mathrm{He}$, we might still suffer the same problems with dryness and consequent opacity.

There have been several previous reports on experimental ocular toxocariasis. Among these, mice, ${ }^{27-29}$ rabbits, ${ }^{28}$ guinea pigs, ${ }^{3031}$ and monkeys ${ }^{32}{ }^{33}$ were evaluated as animal models for this disease. However, none of these 
animals exhibited a high incidence of ocular lesions with a single oral inoculation. Gerbils, on the other hand, showed multiple abnormalities and motile larvae in the retina findings which are comparable in some respects with human cases. ${ }^{18242638}$ Several ophthalmologists have noticed a motile larva in the retina of humans, ${ }^{182639}$ and the ophthalmological findings in those reports are identical to our observations.

Furthermore, in monkeys ${ }^{32}$ and guinea pigs, ${ }^{30}{ }^{31}$ severe pathological changes were only elicited by intravitreal inoculation of live larvae but not by oral inoculation, while gerbils showed severe changes with a single oral inoculation of the eggs.

Eosinophilic granuloma is the most frequent finding with human ocular toxocariasis. ${ }^{35404}$ Although the aetiology of this granuloma is unknown, Watzke reported that the larvae inside a granuloma were still motile after the host had died. ${ }^{11}$ We noticed that larvae in gerbils' fundi migrated vigorously and quickly, suggesting that a local immune response to the motile larvae could not have occurred. We therefore believed that the cellular response would begin when the larvae stopped migrating quickly or after they had died.

We could observe a trace of the larva in real time with an ophthalmoscope. There were two kinds of trace, one which disappeared in 1 day and one which lasted more than 10 days. Several human cases in which a trace of a larva was seen have been reported. ${ }^{18}{ }^{19}$ In one case, the trace remained for more than 2 years in a human eye, ${ }^{19}$ but in our experiment all traces disappeared within 20 days. We can think of three possibilities for the aetiology of the trace: (1) immunological response, (2) direct destruction of retinal layer by larva, and (3) excretory material remaining on the retinal layer. Since these traces were made during migration of a larva, (1) is less likely and (2) and (3) are more likely. If the larvae destroy pigment epithelium, the region may be seen as white to yellowish with an ophthalmoscope, and can be regenerated within 20 days as we observed.

The migrating route of the larvae to the eye is still unclear. This is thought to occur in three ways: (1) through the arteries from the internal carotid artery to the ophthalmic artery, retinal central artery, or ciliary artery; (2) through the brain to the optic nerve; and (3) through the brain to the CSF space then to the choroid. Since choroidal haemorrhage was the most frequent in the early ocular findings and it was often observed simultaneously with motile larva, (3) is suspected to be the most common.

Interestingly, we found vasculitis in orally infected gerbils. These lesions were scattered throughout the peripheral vessels, especially at the site of bifurcation. These data suggested that vasculitis was an immunological event. Although vasculitis was reported in monkeys with an intracarotid injection of $T$ canis larvae, ${ }^{32}{ }^{33}$ this is the first report of the demonstration of vasculitis induced by embryonated eggs of $T$ canis with oral infection. Thus, we believe the gerbil model provides a means of studying the immunological aspects of ocular toxocariasis.

Our study has demonstrated that gerbils can be used as an experimental model for confirming diagnoses, and the aetiology of ocular findings, and applying various treatments for ocular toxocariasis.

The results of this study were presented at the 9th International Congress of Parasitology (24-28 August 1998) in Makuhari, Chiba, Japan.

We thank Professor Masayoshi Iwaki, Department of Ophthalmology, Dr Kazuhiro Yoshikawa, Department of Pathology at Aichi Medical College, and Dr Tetsushi Yasuma of Yasuma Ophthalmology Hospital for their valuable suggestions.

1 Wilder HC. Nematode endophthalmitis. Trans Am Acad Ophthalmol Otolaryngol 1950;55:99-109.

2 Nichols RL. Etiology of visceral larva migrans: I. Diagnostic morphology of infective second toxocaral larvae. F Parasitol morphology of in

3 Irvine WL, Irvine AR Jr. Nematode endophthalmitis. Am $\mathcal{F}$ Ophthalmol 1959;47:185-91.

4 Ashton N. Communications-larval granulomatousis of the retina due to Toxocara. Br f Ophthalmol 1960;44:129-48. 5 Duguid IM. Chronic endophthalmitis due to Toxocara. $\mathrm{Br} F$ Ophthalmol 1961;45:787-96.

6 Yoshioka H. Nematode endophthalmitis, possibly due to Toxocara canis. Report of a case. $f$ Clin Ophthalmol 1966;20:605-10

7 Rey A. Nematode endophthalmitis due to Toxocara. $\mathrm{Br} f$ Ophthalmol 1962;46:616-8.

8 Wilkinson $\mathrm{CP}$, Welch RB. Intraocular toxocara. Am $\mathcal{f}$ Ophthalmol 1971;71:921-30.

9 Maguire AM, Green WR, Michels RG, et al. Recovery of intraocular Toxocara canis by pars plana vitrectomy. intraocular Toxocara canis by

10 Glickman LT, Shantz PM. Epidemiology and pathogenesis of zoonotic toxocariasis. Epidemiol Rev 1981;3:230-50.

11 Watzke RC. Ocular Toxocara canis infection: clinical and experimental features. Trans New Orleans Acad Ophthalmol 1983;31:263-71.

12 Searl SS, Moazed K, Albert DM, et al. Ocular toxocariasis presenting as leukocoria in a patient with low ELISA titer to Toxocara canis. Ophthalmology 1981;88:1302-6.

13 Pollard ZF. Long-term follow-up in patients with ocular toxocariasis as measured by ELISA titers. Ann Ophthalmol 1987;19:167-9.

14 Kielar RA. Toxocara canis endophthalmitis with low ELISA titer. Ann Ophthalmol 1983;15:447-9.

15 Delcastillo J, Herreros G, Guillen JL, et al. Bilateral ocular toxocariasis demonstrated by aqueous humor enzymelinked immunosorbent assay. Am f Ophthalmol 1995;119: 514-16.

16 Glickman L, Cypess R, Hiles D, et al. Toxocara-specific antibody in the serum and aqueous humor of a patient with presumed ocular and visceral toxocariasis. Am $\mathcal{F}$ Trop Med Hyg 1979;28:29-35.

17 Brasseur G, Charlin JF, Brasseur $\mathrm{P}$, et al. Ocular toxocariasis; diagnostic and therapeutic attainments [in toxocariasis; diagnostic and therapeutic

18 Fitzgerald CR, Rubin ML, Fla G. Intraocular parasite destroyed by photocoagulation. Arch Ophthalmol 1974;91: $162-4$.

19 Sorr EM. Meandering ocular toxocariasis. Retina 1984;4: 90-6.

20 Siam AL. Toxocaral chorio-retinitis. Treatment of early cases with photocoagulation. $\mathrm{Br} \mathcal{F}$ Ophthalmol 1973;57: $700-3$.

21 Gass JD, Braunstein RA. Further observations concerning the diffuse unilateral subacute neuroretinitis syndrome. Arch Ophthalmol 1983;101:1689-97.

22 Rodriguez A. Early pars plana vitrectomy in chronic endophthalmitis of toxocariasis. Graefes Arch Clin Exp Ophthalmol 1986;224:218-20.

23 Belmont JB, Irvine A, Benson W, et al. Vitrectomy in ocular toxocariasis. Arch Ophthalmol 1982;100:1912-15.

24 Matri L, Ghorbal M, Ayadi A, et al. Toxocara canis in apparently bilateral ocular site. $\mathcal{f}$ Fr Ophtalmol 1990;13: 303-8.

25 Iwaki M. Cryotherapy for ocular toxocariasis. $f_{p n} \mathcal{f}$ Clin Ophthalmol 1993;47:537-40.

26 Byers B, Kimura SJ. Uveitis after death of a larva in the vitreous cavity. Am $\mathcal{F}$ Ophthalmol 1974;77:63-6.

27 Ghafoor SY, Smith HV, Lee WR, et al. Experimental ocular toxocariasis: a mouse model. Br F Ophthalmol 1984;68:8996.

28 Kunishige A. Histologic and histochemical studies on experimental "visceral larva migrans" in rabbits and mice [in Japanese]. Shikoku Acta Medica 1964;21:546-67

29 Olson LJ. Ocular toxocariasis in mice: distribution of larvae and lesions. Int $\mathcal{F}$ Parasitol 1976;6:2471.

30 John T, Donnelly JJ, Rockey JH. Experimental ocular Toxocara canis and Ascaris suum infection: in vivo and in vitro study. Trans Pa Acad Ophthalmol Otolaryngol 1983;36:1317.

31 Miyamoto K. Experimental toxocariasis in abnormal hosts. 2) Histophotological studies on mice and guinea pigs infected with Toxocara canis. Fpn f Parasitol 1972; 21(suppl):54 
32 Watzke RC, Oaks JA, Folk JC. Toxocara canis infection of the eye. Correlation of clinical observations with developing pathology in the primate model. Arch Ophthalmol 1984 102:282-91.

33 Luxenberg MN. An experimental approach to the study of intraocular Toxocara canis. Trans Am Ophthalmol Soc 1979; 77:542-602.

34 Klei TR, McVay CS, Dennis VA, et al. Brugia pahangi: effects of duration of infection and parasite burden on lymphatic lesion severity, granulomatous hypersensitivity, and immune responses in jirds (Meriones unguiculatus). Exp Parasitol 1990;71:393-405

35 Maruyama H, Kobayashi T, Tsuchiya K, et al. Sensitive enzyme-linked imunosorbent assay (ELISA) method to measure parasite-specific antibodies of Mongolian gerbils. fpn f Parasitol 1994;43:351-7.

36 Campbell D, Chadee K. Interleukin (IL)-2, IL-4, and tumor necrosis factor- $\beta$ responses during Entamoeba histolytica liver abscess development in gerbils. F Infect Dis 1997;175: 1176-83.

37 Oshima T. Standardization of techniques for infecting mice with Toxocara canis and observations on the normal migration routes of the larvae. 7 Parasitol 1961;47:652-6.

38 Gillespie SH, Dinning WJ, Voller A, et al. The spectrum of ocular toxocariasis. Eye 1993; 7:415-8.

39 Price JA Jr, Tenn J, Wadsworth JAC, et al. An intraretinal worm. Report of a case of macular retinopathy caused by invasion of the retina by a worm. Arch Ophthalmol 1970;83: 768-70.

40 Rey A. Nematode endophthalmitis due to Toxocara. Br $\mathcal{F}$ Ophthalmol 1962;46:616-8.

41 Harris W. Pseudo-glioma due to larval choroido-retinal granulomatosis. Br F Ophthalmol 1961;45:144-6. 